Article

\title{
Downlink Spectral Efficiency Analysis in Distributed Massive MIMO with Phase Noise
}

\author{
Qian Lv ${ }^{(D)}$, Jiamin Li *, Pengcheng Zhu, Dongming Wang and Xiaohu You
}

National Mobile Communications Research Laboratory, Southeast University, Nanjing 210096, China; seulvqian@seu.edu.cn (Q.L.); p.zhu@seu.edu.cn (P.Z.); wangdm@seu.edu.cn (D.W.); xhyu@seu.edu.cn (X.Y.)

* Correspondence: lijiamin@seu.edu.cn; Tel.: +86-025-5209-1635

Received: 25 October 2018 ; Accepted:10 November 2018 ; Published: 12 November 2018

\begin{abstract}
To achieve the advantages provided by massive multiple-input multiple-output (MIMO), a large number of antennas need to be deployed at the base station. However, for the reason of cost, inexpensive hardwares are employed in the realistic scenario, which makes the system distorted by hardware impairments. Hence, in this paper, we analyze the downlink spectral efficiency in distributed massive MIMO with phase noise and amplified thermal noise. We provide an effective channel model considering large-scale fading, small-scale fast fading and phase noise. Based on the model, the estimated channel state information (CSI) is obtained during the pilot phase. Under the imperfect CSI, the closed-form expressions of downlink achievable rates with maximum ratio transmission (MRT) and zero-forcing (ZF) precoders in distributed massive MIMO are derived. Furthermore, we also give the user ultimate achievable rates when the number of antennas tends to infinity with both precoders. Based on these expressions, we analyze the impacts of phase noise on the spectral efficiency. It can be concluded that the same limit rate is achieved with both precoders when phase noise is present, and phase noise limits the spectral efficiency. Numerical results show that ZF outdoes MRT precoder in spectral efficiency and ZF precoder is more affected by phase noise.
\end{abstract}

Keywords: distributed massive MIMO; phase noise; amplified thermal noise; spectral efficiency

\section{Introduction}

Massive multiple-input multiple-output (MIMO) is becoming a promising technology to provide significant gains [1-6]. Since it was first proposed, massive MIMO has been studied extensively. The main feature of massive MIMO is that hundreds (or even thousands) of antennas are employed at each base station, simultaneously serving tens of users in the same time-frequency resource, which offers big advantages compared to conventional MIMO. Firstly, it can bring unprecedented spatial degrees-of-freedom, which enables the improvement of spectral efficiency and energy efficiency even with simple linear receivers or precoders [7]. In addition, user channels in massive MIMO systems are nearly orthogonal and fast fading, intra-cell interference can be averaged out. Massive MIMO can be divided into two categories: one is co-located massive MIMO and the other is distributed massive MIMO [8]. The latter has promising advantages of increasing energy efficiency, system coverage and spectral efficiency, which results from the increase in macro-diversity gain and the reduction in access distance [9-15]. Considering these advantages, we analyze the spectral efficiency of distributed massive MIMO in this paper. Notably, due to the different access distance between each user and all remote antenna units (RAUs), the channel vectors are non-isotropic, which makes the analysis of performance in distributed massive MIMO more difficult and more complex.

In practical communication systems, inevitable hardware impairments occur and cannot be eliminated even after applying calibration and compensation techniques $[16,17]$. These impairments can be divided into two categories: multiplicative distortion and additive distortion. Phase noise 
introduced by the local oscillators of transceivers is the multiplicative distortion. It will cause random rotations of the transmitted data symbols, which degrades the system performance. Furthermore, phase noise makes the estimated channel state information (CSI) more inaccurate and it introduces a phenomenon called channel aging which means the estimated CSI obtained during pilot phase is different from that used for downlink transmission. It is pointed out in [8] that the deployment cost and circuit power consumption of massive MIMO scale linearly with the number of antennas. Therefore inexpensive but hardware-constraint hardware may be deployed for the reason of cost, which makes the hardware impairments more severe in massive MIMO.

Analyzing the spectral efficiency is a fundamental method to evaluate the impacts of phase noise. The impacts of phase noise for uplink transmission have been studied in [18-21] and for downlink transmission were investigated in [22-24]. The impacts of phase noise on physical layer security for downlink massive MIMO were investigate in [22]. The achievable rate was derived in [23] considering the frequency-selective multipath fading channel. The capacity of downlink transmission with linear precoders was analyzed in [24] but it assumed that the number of antennas and users was asymptotically large and it only considered a co-located MIMO system.

Herein, considering a distributed massive MIMO with phase noise and amplified thermal noise, we analyzed the downlink spectral efficiency for any number of antennas and users. Followings are the key contributions of this paper:

1. In distributed massive MIMO, the channel vectors are non-isotropic and the correlation between channel vectors and intended beams for each user are destroyed by phase noise. Hence, we first obtain the distributions of the desired signal and interference powers, which is challenging and complex.

2. Considering both zero-forcing (ZF) and maximum ratio transmission (MRT) precoders, we obtain the closed-form expressions of the downlink ergodic achievable rates with imperfect CSI and hardware impairments in distributed massive MIMO. These closed-form expressions are accurate for any number of antennas and users in both distributed massive MIMO and co-locate massive MIMO. Furthermore, they are derived under imperfect CSI which is more realistic, and they enable the analysis of performance degradation caused by phase noise.

3. The ultimate achievable rate per user is obtained when the number of antennas per remote antenna unit (RAU) goes infinity. It can be used to investigate the asymptotic performance of distributed massive MIMO with hardware impairments.

4. The theoretical results are verified by Monte Carlo simulations, and we have a deep insight into the impacts of phase noise.

The rest of this paper is organized as follows. System model including system configuration, a model describing phase noise and an effective channel model is introduced in Section 2. We obtain the estimated CSI during the uplink pilot training phase and analyze the spectral efficiency with linear precoders in Section 3. Numerical results are given in Section 4. A conclusion is provided in Section 5.

Notation: Column vectors $\mathbf{x}$ and matrices $\mathbf{X}$ are denoted by bold letters in lower case and in upper case, respectively. $\mathbf{I}_{N}$ is a $N \times N$ identity matrix. $(\cdot)^{\mathrm{H}}$ and $(\cdot)^{\mathrm{T}}$ are the conjugate transpose and transpose operator, respectively. Scalars $x$ are denoted by italic letters. $|x|$ represents the absolute value of $x$ and $\|\mathbf{X}\|$ denotes the spectral norm of $\mathbf{X} . \mathbb{E}[\cdot]$ and $\operatorname{var}(\cdot)$ represent the expectation operator and variance operator, respectively. $\mathcal{C N}\left(0, \sigma^{2}\right)$ represents circularly symmetric complex Gaussian distribution with mean zero and variance $\sigma^{2} . \Gamma(k, \theta)$ means Gamma distribution with shape parameter $k$ and scale parameter $\theta$. Similarly, $\operatorname{Nakagami}(m, \Omega)$ means Nakagami distribution with shape parameter $m$ and controlling spread parameter $\Omega$.

\section{System Model}

Considering a distributed massive MIMO system, we first describe the system configuration and give the conventional channel model. Next, we present a model describing phase noise and give an effective channel model incorporating the impacts of phase noise. 
We consider the downlink transmission of a single-cell multi-user distributed massive MIMO system comprising $M$ RAUs and $K$ single-antenna users as in Figure 1. Each RAU is equipped with an array of $N$ antennas. All users and RAUs are randomly distributed in the cell.
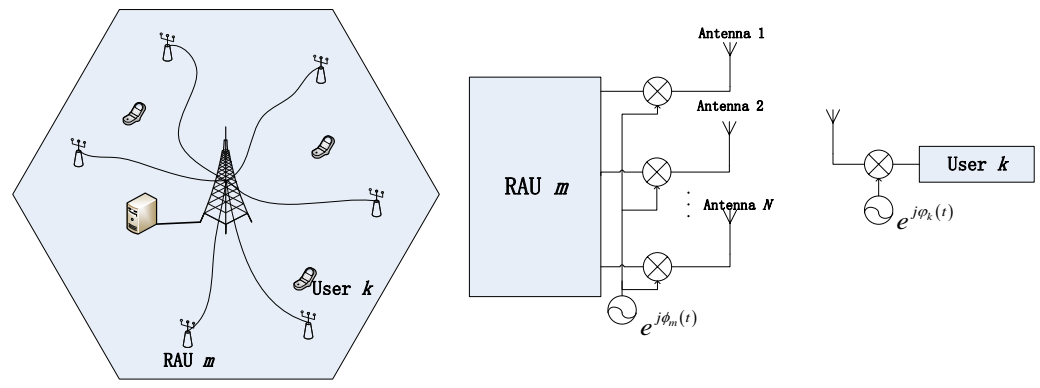

Figure 1. System Model.

Frequency-flat fading channels are assumed and the system runs in time-division duplex (TDD) protocol. The channel vector between all RAUs and the $k$-th user is given by

$$
\tilde{\mathbf{g}}_{k} \triangleq\left[\tilde{g}_{k}^{1} \cdots \tilde{g}_{k}^{M N}\right]=\boldsymbol{\Lambda}_{k}^{1 / 2} \mathbf{h}_{k}
$$

where $\boldsymbol{\Lambda}_{k}=\mathbb{E}\left[\tilde{\mathbf{g}}_{k} \tilde{\mathbf{g}}_{k}^{\mathrm{H}}\right]=\operatorname{diag}\left(\lambda_{1, k} \cdots \lambda_{M, k}\right) \otimes \mathbf{I}_{N}$ is the covariance matrix, $\lambda_{m, k} \triangleq c d_{m, k}^{-\alpha}$ denotes the path loss between the $m$-th RAU and the $k$-th user, $d_{m, k}$ is the corresponding distance, $\alpha$ is the path loss exponent, $c$ is the median of the mean path gain at a reference distance $d_{m, k}=1 \mathrm{~km}$, and $\mathbf{h}_{k} \sim \mathcal{C N}\left(0, \mathbf{I}_{M N}\right)$ is the small-scale fast fading vector.

In this paper, we consider a more realistic scenario where the antennas deployed at each RAU are inexpensive and hardware-constrained. Specifically, each antenna experiences phase noise which distorts communication. The phase noise means the multiplicative phase drift in the signal, which comes from the local oscillators (LOs) of the RAUs and users. We assume that the LOs are free-running without a phase-locked loop (PLL), and then the phase noise is commonly modeled as a discrete-time independent Wiener process $[8,25]$. Mathematically, the phase noises at the LOs of the $n$-th antenna and the $k$-th user are denoted as

$$
\begin{aligned}
& \phi_{n}(t) \sim \mathcal{N}\left(\phi_{n}(t-1), \sigma_{\phi, n}^{2}\right), \\
& \varphi_{k}(t) \sim \mathcal{N}\left(\varphi_{k}(t-1), \sigma_{\varphi, k}^{2}\right),
\end{aligned}
$$

which equal the previous realization $\phi_{n}(t-1)$ and $\varphi_{k}(t-1)$ plus an independent zero-mean Gaussian random increment with variances $\sigma_{\phi, n}^{2}$ and $\sigma_{\phi, k}^{2}$. The variances are dependent on the carrier frequency and symbol time [25].

The phase noise can be independent or correlated between antennas of each RAU. In our analysis, we have assumed that the phase noise correlated between antennas of one RAU and independent among RAUs. Then by expressing the total phase noise as a multiplicative factor, we can rewrite the channel vector as

$$
\mathbf{g}_{k}(t)=\boldsymbol{\Theta}_{k}(t) \tilde{\mathbf{g}}_{k}
$$

where $\boldsymbol{\Theta}_{k}(t) \triangleq \operatorname{diag}\left(e^{j \theta_{k}^{1}(t)}, \ldots, e^{j \theta_{k}^{M N}(t)}\right)=e^{j \varphi_{k}(t)} \mathbf{\Phi}(t) \in \mathbb{C}^{M N \times M N}$ is the total phase noise, wherein $\boldsymbol{\Phi}(t) \triangleq \operatorname{diag}\left(e^{j \phi_{1}(t)}, \ldots, e^{j \phi_{M}(t)}\right) \otimes \mathbf{I}_{N}$ is the phase noise induced by all RAUs, and similarly, $e^{j \varphi_{k}(t)}$ corresponds to the phase drift pruduced by the $k$-th user. Notably, because of the presence of phase noise, the effective channel becomes time-dependent. 
Remark 1. The conventional channel model without phase noise is obtained when $\sigma_{\phi, n}^{2}=\sigma_{\varphi, k}^{2}=0, \forall n, k$.

\section{Downlink Spectral Efficiency Analysis}

In this section, firstly, based on the effective channel model given above, we assume pilot sequence aided transmission is employed and give the channel estimation. Next, since the channel vectors are non-isotropic in distributed massive MIMO and the correlation between channel vectors and intended beams for each user is destroyed by phase noise, we give the approximated distribution of desired signal and interference powers. After that, we derive the closed-form expressions of the ergodic achievable downlink rates with both MRT and ZF precoders.

\subsection{Channel Estimation}

As mentioned before, the transmission protocol is assumed as TDD. Each coherence block occupying $T$ channel uses is split into two parts: one for uplink pilot symbols and the other for downlink data symbols. In order to guarantee that the pilot symbols of $K$ users are orthogonal to each other, it's necessary to allocate $\tau \geq K$ symbols for pilot transmission. Then the remaining $T-\tau$ channel uses are used for downlink data transmission.

During the pilot training phase, the pilot sequence $\mathbf{x}_{k} \triangleq\left[x_{k}(1), \cdots, x_{k}(\tau)\right]^{\mathrm{T}}$ is assigned to user $k$. Incorporating the hardware impairments, the received pilot vector $\mathbf{y}_{\mathrm{p}}$ at the base station at time $t \in[0, \tau]$ is given as

$$
\mathbf{y}_{\mathbf{p}}(t)=\sum_{k=1}^{K} \mathbf{g}_{k}(t) x_{k}(t)+\mathbf{n}^{\mathrm{BS}}(t),
$$

where $\mathbf{n}^{\mathrm{BS}}(t) \sim \mathcal{C N}\left(0, \xi^{\mathrm{BS}} \mathbf{I}_{M N}\right)$ is the amplified thermal noise at time slot $t$, and its variance $\xi^{\mathrm{BS}}$ is larger than the variance $\sigma^{2}$ of thermal noise. This is because of the effects of low noise amplifiers, mixers and other components.

Let $\mathbf{\Psi} \triangleq\left[\mathbf{y}_{\mathrm{p}}^{\mathrm{T}}(1), \cdots, \mathbf{y}_{\mathrm{p}}^{\mathrm{T}}(\tau)\right]^{\mathrm{T}} \in \mathbb{C}^{\tau M N \times 1}$. Motivated by $[8,26]$, the Linear Minimum Mean Square Error (LMMSE) estimation of the channel of the $k$-th user obtained by pilot training is given by

$$
\hat{\mathbf{g}}_{k}(t)=\boldsymbol{\Lambda}_{k} \mathbf{H}_{k}(t) \boldsymbol{\Sigma}^{-1} \mathbf{\Psi},
$$

where

$$
\begin{aligned}
\mathbf{H}_{k}(t) & =\left[\mathbf{H}_{k, 1}(t), \cdots, \mathbf{H}_{k, \tau}(t)\right], \\
\Sigma & \triangleq \sum_{j=1}^{K} \mathbf{B}_{j}+\xi^{\mathrm{BS}} \mathbf{I}_{\tau M N}, \\
\mathbf{H}_{k, i}(t) & =x_{k}^{*}(i) \mathbf{D}_{k, i}(t), \\
\mathbf{D}_{k, i}(t) & =\operatorname{diag}\left(e^{-\frac{\sigma_{\phi, 1}^{2}+\sigma_{\varphi, k}^{2}}{2}|t-i|}, \ldots, e^{-\frac{\sigma_{\phi, M}^{2}+\sigma_{\varphi, k}^{2}}{2}|t-i|}\right) \otimes \mathbf{I}_{N}, \\
{\left[\mathbf{B}_{j}\right]_{u, v} } & \triangleq \Lambda_{j} x_{j}(u) x_{j}^{*}(v) \operatorname{diag}\left(e^{-\frac{\sigma_{\phi, 1}^{2}+\sigma_{\varphi, j}^{2}}{2}|u-v|}, \ldots, e^{-\frac{\sigma_{\phi, M}^{2}+\sigma_{\varphi, j}^{2}}{2}|u-v|}\right) \otimes \mathbf{I}_{N} .
\end{aligned}
$$

The pilot sequences can be designed in different ways. Without loss of generation, in this paper we assume that the number of pilot symbols is equal to that of users, i.e., $\tau=K$. More specifically, we assume that the set of orthogonal pilot sequences $\mathbf{X}_{\mathrm{P}} \triangleq\left[\mathbf{x}_{1}, \cdots, \mathbf{x}_{K}\right]$ is a diagonal matrix and each element of it is $\sqrt{\rho_{\mathrm{p}}}$, wherein $\rho_{\mathrm{p}}$ is the average transmit power of pilot symbols. This is equivalent to the assumption made in $[18,20]$. 
Under these assumptions, we give a definition of

$$
\beta_{m, k}(t)=\frac{e^{-\frac{\sigma_{\varphi, k}^{2}+\sigma_{\phi, m}^{2}}{2}|t-k|} \sqrt{\rho_{\mathrm{p}}} \lambda_{m, k}}{\sqrt{\rho_{\mathrm{p}} \lambda_{m, k}+\xi^{\mathrm{BS}}}},
$$

then we can rewrite the LMMSE estimation $\hat{\mathbf{g}}_{k}(t)$ in (6) as

$$
\begin{aligned}
\hat{\mathbf{g}}_{k}(t) & =\boldsymbol{\Lambda}_{k} \mathbf{H}_{k}(t) \boldsymbol{\Sigma}^{-1 / 2} \hat{\mathbf{h}}_{k} \\
& =\left(\beta_{1, k}(t) \hat{\mathbf{h}}_{1, k}^{\mathrm{T}}, \cdots, \beta_{M, k}(t) \hat{\mathbf{h}}_{M, k}^{\mathrm{T}}\right)^{\mathrm{T}},
\end{aligned}
$$

where $\beta_{m, k}(t)$ is the equivalent large-scale fading part from user $k$ to RAU $m$ and $\hat{\mathbf{h}}_{k}=\left[\hat{\mathbf{h}}_{1, k}^{\mathrm{T}}, \cdots, \hat{\mathbf{h}}_{M, k}^{\mathrm{T}}\right]^{\mathrm{T}}=\boldsymbol{\Sigma}^{-1 / 2} \mathbf{\Psi} \sim \mathcal{C N}\left(0, \mathbf{I}_{M N}\right)$ represents the equivalent small-scale fast fading part.

Because of the orthogonality principle of LMMSE estimation theory, the channel vector $\mathbf{g}_{k}(t)$ can be decomposed as

$$
\mathbf{g}_{k}(t)=\hat{\mathbf{g}}_{k}(t)+\mathbf{e}_{k}(t),
$$

where $\mathbf{e}_{k}(t)$ is the uncorrelated and statistically independent of $\hat{\mathbf{g}}_{k}(t)$ estimation error.

During the pilot transmission phase, we obtain the estimated channel showing in (8). In our analysis, it is assumed that the beamforming vector is designed by using the estimated CSI once during the pilot transmission phase and then is applied for the entire duration of the downlink transmission phase.

\subsection{Downlink Signal Model}

For downlink transmission, the received signal of user $k$ at time $t \in[\tau+1, T]$ is given as

$$
r_{k}(t)=\sqrt{\rho_{\mathrm{dl}}} \tilde{\mathbf{g}}_{k}^{\mathrm{H}} \Theta_{k}^{*}(t) \mathbf{x}+n^{\mathrm{UE}}(t),
$$

where $\rho_{\mathrm{dl}}$ is the downlink transmission power, $n^{\mathrm{UE}}(t) \sim \mathcal{C N}\left(0, \xi^{\mathrm{UE}}\right)$ is the amplified thermal noise of users at time slot $t, \xi^{\mathrm{UE}}$ is the variance of the noise, and $\mathbf{x} \in \mathbb{C}^{M N \times 1}$ is the signal vector transmitted by all $M$ RAUs. Specifically, $\mathbf{x}$ can be given by

$$
\mathbf{x}=\sum_{l=1}^{K} \mathbf{w}_{l} s_{l},
$$

where $s_{l} \sim \mathcal{C N}(0,1)$ is the transmitted data symbol assigned for user $l$, and $\mathbf{w}_{l}$ is the beamforming vector designed at time slot $\tau$. MRT and ZF precoders are considered in our analysis. Mathematically, these two linear precoders can be defined as

$$
\mathbf{w}_{l}= \begin{cases}\frac{\hat{\mathbf{g}}_{l}(\tau)}{\left\|\hat{\mathbf{s}}_{l}(\tau)\right\|} & \mathrm{MRT}, \\ \mathbf{a}_{l}(\tau) & \mathrm{ZF} \\ \left\|\mathbf{a}_{l}(\tau)\right\| & \end{cases}
$$

where $\mathbf{a}_{l}(\tau)$ is the $l$-th column of $\hat{\mathbf{G}}(\tau)\left(\hat{\mathbf{G}}^{\mathrm{H}}(\tau) \hat{\mathbf{G}}(\tau)\right)^{-1}$, wherein $\hat{\mathbf{G}}(\tau)=\left[\hat{\mathbf{g}}_{1}(\tau), \cdots, \hat{\mathbf{g}}_{K}(\tau)\right]$. Considering (4), we can rewrite (10) as

$$
r_{k}(t)=\sqrt{\rho_{\mathrm{dl}}} \mathbf{g}_{k}^{\mathrm{H}}(\tau) \tilde{\Theta}_{k}(t) \mathbf{x}+n^{\mathrm{UE}}(t),
$$

where

$$
\tilde{\Theta}_{k}(t) \triangleq \operatorname{diag}\left(e^{-j\left(\theta_{k}^{1}(t)-\theta_{k}^{1}(\tau)\right)}, \cdots, e^{-j\left(\theta_{k}^{M N}(t)-\theta_{k}^{M N}(\tau)\right)}\right) .
$$


It is assumed that users have the statistical properties of the channel and they don't carry out channel estimation. So only statical CSI can be used by downlink users to detect the signal. Motivated by [27], we rewrite the received data as

$$
r_{k}(t)=\sqrt{\rho_{\mathrm{dl}} \mathbb{E}}\left[\mathbf{g}_{k}^{\mathrm{H}}(\tau) \tilde{\Theta}_{k}(t) \mathbf{w}_{k}\right] s_{k}+n^{\prime},
$$

where

$$
n^{\prime}=\sqrt{\rho_{\mathrm{dl}}}\left(\mathbf{g}_{k}^{\mathrm{H}}(\tau) \tilde{\Theta}_{k}(t) \mathbf{w}_{k}-\mathbb{E}\left[\mathbf{g}_{k}^{\mathrm{H}}(\tau) \tilde{\Theta}_{k}(t) \mathbf{w}_{k}\right]\right) s_{k}+\sqrt{\rho_{\mathrm{dl}}} \sum_{i \neq k}^{K} \mathbf{g}_{k}^{\mathrm{H}}(\tau) \tilde{\Theta}_{k}(t) \mathbf{w}_{i} s_{i}+n^{\mathrm{UE}}(t) .
$$

Suppose $\mathbb{E}\left[\mathbf{g}_{k}^{\mathrm{H}}(\tau) \tilde{\Theta}_{k}(t) \mathbf{w}_{k}\right] s_{k}$ is the only signal needed at user $k$, and treating $n^{\prime}$ as unrelated Gaussian distributed additive noise [28,29], the achievable downlink rate of user $k$ is denoted as

$$
R_{k}=\log _{2}\left(1+\frac{\rho_{\mathrm{dl}}\left|\mathbb{E}\left[\mathbf{g}_{k}^{\mathrm{H}}(\tau) \tilde{\Theta}_{k}(t) \mathbf{w}_{k}\right]\right|^{2}}{A(t)+B(t)+\xi^{\mathrm{UE}}}\right)
$$

where

$$
\begin{aligned}
& A(t)=\rho_{\mathrm{dl}} \operatorname{var}\left(\mathbf{g}_{k}^{\mathrm{H}}(\tau) \tilde{\Theta}_{k}(t) \mathbf{w}_{k}\right) \\
& B(t)=\sum_{i \neq k}^{K} \rho_{\mathrm{dl}} \mathbb{E}\left[\left|\mathbf{g}_{k}^{\mathrm{H}}(\tau) \tilde{\Theta}_{k}(t) \mathbf{w}_{i}\right|^{2}\right]
\end{aligned}
$$

\subsection{Downlink Achievable Rates}

From (16) we can find that the correlation between $\mathbf{g}_{k}$ and $\mathbf{w}_{k}$ are destroyed by phase noise and the powers of non-isotropic channel vectors projected onto the precoder subspace are necessary to obtain the closed-form expressions. Hence we first present some preliminary lemmas which help us to obtain the approximated and isotropic results.

Lemma 1 ([28]). For an isotropic random vector $\mathbf{x} \in \mathbb{C}^{N \times 1}$ whose elements are independent and all distributed as $\mathcal{C N}\left(0, \sigma^{2}\right)$, then the distribution of $\mathbf{x}^{\mathrm{H}} \mathbf{x}$ is $\Gamma\left(N, \sigma^{2}\right)$.

The strength of the estimated channel from user $k$ to all RAUs is

$$
\hat{\mathbf{g}}_{k}^{\mathrm{H}}(t) \hat{\mathbf{g}}_{k}(t)=\sum_{m=1}^{M} \beta_{m, k}^{2}(t) \hat{\mathbf{h}}_{m, k}^{\mathrm{H}} \hat{\mathbf{h}}_{m, k}
$$

According to Lemma $1, \beta_{m, k}^{2}(t) \hat{\mathbf{h}}_{m, k}^{\mathrm{H}} \hat{\mathbf{h}}_{m, k}$ is distributed as $\Gamma\left(N, \beta_{m, k}^{2}(t)\right)$. Hence (17) is the sum of $M$ non-identically distributed but independent items. To obtain its distribution, Lemma 2 stated bellow can be used.

Lemma 2 ([15]). If $\left\{x_{i}\right\}$ are a set of random variables and independent of each other, each term is distributed as $\Gamma\left(\chi_{i}, \theta_{i}\right)$. Then the distribution of the sum $\sum_{i} x_{i}$ can be approximated as $\sum_{i} X_{i} \sim \Gamma(\chi, \theta)$ wherein

$$
\chi=\frac{\left(\sum_{i} \chi_{i} \theta_{i}\right)^{2}}{\sum_{i} \chi_{i} \theta_{i}^{2}}, \theta=\frac{\sum_{i} \chi_{i} \theta_{i}^{2}}{\sum_{i} \chi_{i} \theta_{i}}
$$


Remark 2. From Lemma 2, the distribution of $\hat{\mathbf{g}}_{k}^{\mathrm{H}}(t) \hat{\mathbf{g}}_{k}(t)$ can be approximated as $\Gamma\left(\chi_{k}(t), \theta_{k}(t)\right)$, wherein

$$
\begin{aligned}
& \chi_{k}(t)=N \frac{\left(\sum_{m=1}^{M} \beta_{m, k}^{2}(t)\right)^{2}}{\sum_{m=1}^{M} \beta_{m, k}^{4}(t)}, \\
& \theta_{k}(t)=\frac{\sum_{m=1}^{M} \beta_{m, k}^{4}(t)}{\sum_{m=1}^{M} \beta_{m, k}^{2}(t)} .
\end{aligned}
$$

Similarly, we can also give the distribution of $\mathbf{e}_{k}^{\mathrm{H}}(t) \mathbf{e}_{k}(t)$ as $\Gamma\left(\chi_{\mathrm{e}}(t), \theta_{\mathrm{e}}(t)\right)$, wherein

$$
\begin{aligned}
& \chi_{\mathrm{e}}(t)=N \frac{\left(\sum_{m=1}^{M} \eta_{m, k}^{2}(t)\right)^{2}}{\sum_{m=1}^{M} \eta_{m, k}^{4}(t)} \\
& \theta_{\mathrm{e}}(t)=\frac{\sum_{m=1}^{M} \eta_{m, k}^{4}(t)}{\sum_{m=1}^{M} \eta_{m, k}^{2}(t)}
\end{aligned}
$$

where $\eta_{m, k}^{2}(t)=\lambda_{m, k}-\beta_{m, k}^{2}(t)$.

Based on the lemmas and analysis above, we give the following lemma about the projection principle of non-isotropic vectors.

Lemma 3 ([28]). When we project an MN-dimensional non-isotropic estimated channel vector $\hat{\mathbf{g}}_{k} \in \mathbb{C}^{M N \times 1}$ onto a p-dimensional subspace, we can give the approximated distribution of the projection power as $\Gamma\left(p \chi_{k} /(M N), \theta_{k}\right)$.

Remark 3. The dimension $p$ can be given by $p=M N$ with MRT precoder and $p=M N-K+1$ with ZF precoder, respectively, and for any independent beam, we can have $p=1[30,31]$.

When MRT and ZF precoders are employed, based on the analysis above, we can give the distribution of the signal power at user $k$ and the distribution of the interference power at user $k$

$$
\begin{aligned}
& \left|\hat{\mathbf{g}}_{k}^{\mathrm{H}}(\tau) \frac{\hat{\mathbf{g}}_{k}(\tau)}{\left\|\hat{\mathbf{g}}_{k}(\tau)\right\|}\right|^{2} \sim \Gamma\left(\chi_{k}(\tau), \theta_{k}(\tau)\right), \\
& \left|\hat{\mathbf{g}}_{k}^{\mathrm{H}}(\tau) \frac{\mathbf{a}_{k}(\tau)}{\left\|\mathbf{a}_{k}(\tau)\right\|}\right|^{2} \sim \Gamma\left(\frac{M N-K+1}{M N} \chi_{k}(\tau), \theta_{k}(\tau)\right), \\
& \left|\mathbf{e}_{k}^{\mathrm{H}}(\tau) \mathbf{w}_{i}\right|^{2} \sim \Gamma\left(\frac{1}{M N} \chi_{\mathrm{e}}(\tau), \theta_{\mathrm{e}}(\tau)\right) .
\end{aligned}
$$

Notably, $\mathbf{w}_{i}$ in (25) can be either MRT precoder or ZF precoder and the equation still holds when $i=k$, due to the independence of $\mathbf{e}_{k}(t)$ and $\mathbf{w}_{i}$.

Based on the analysis above, we can give the approximated distribution of $\left|\hat{\mathbf{g}}_{k}^{\mathrm{H}}(\tau) \tilde{\Theta}_{k}(t) \mathbf{w}_{k}\right|^{2}$ as

$$
\begin{aligned}
& \left|\hat{\mathbf{g}}_{k}^{\mathrm{H}}(\tau) \tilde{\Theta}_{k}(t) \frac{\hat{\mathbf{g}}_{k}(\tau)}{\left\|\hat{\mathbf{g}}_{k}(\tau)\right\|}\right|^{2} \sim \Gamma\left(\chi_{k}(\tau), \theta_{k}(\tau)\right), \\
& \left|\hat{\mathbf{g}}_{k}^{\mathrm{H}}(\tau) \tilde{\Theta}_{k}(t) \frac{\mathbf{a}_{k}(t)}{\left\|\mathbf{a}_{k}(t)\right\|}\right|^{2} \sim \Gamma\left(\frac{M N-K+1}{M N} \chi_{k}(t), \theta_{k}(t)\right) .
\end{aligned}
$$

Figure 2 verifies the accuracy of the approximation in (26) and (27). It illustrates the cumulative distribution function (CDF) curves of $\left|\hat{\mathbf{g}}_{k}^{\mathrm{H}}(\tau) \tilde{\Theta}_{k}(t) \mathbf{w}_{k}\right|^{2}$ with MRT precoder. The phase noise variance is set as $\sigma_{\phi}^{2}=\sigma_{\varphi}^{2}=10^{-2}$. It can be seen that although the random variable $\tilde{\Theta}_{k}(t)$ will destroy the correlation of $\hat{\mathbf{g}}_{k}$ and $\mathbf{w}_{k}$, the approximation is exactly accurate when the variance of the phase noise is $10^{-2}$, and it will be more accurate when the variance of the phase noise is lower $10^{-2}$. 
For ZF precoder, we can get the same conclusion. Meanwhile, the phase noise variance is generally $\sigma_{\phi}^{2}=\sigma_{\varphi}^{2}=1.58 \times 10^{-4}[8,20,26]$. Hence, it's reasonable to use (26) and (27) to analyze the downlink spectral efficiency.

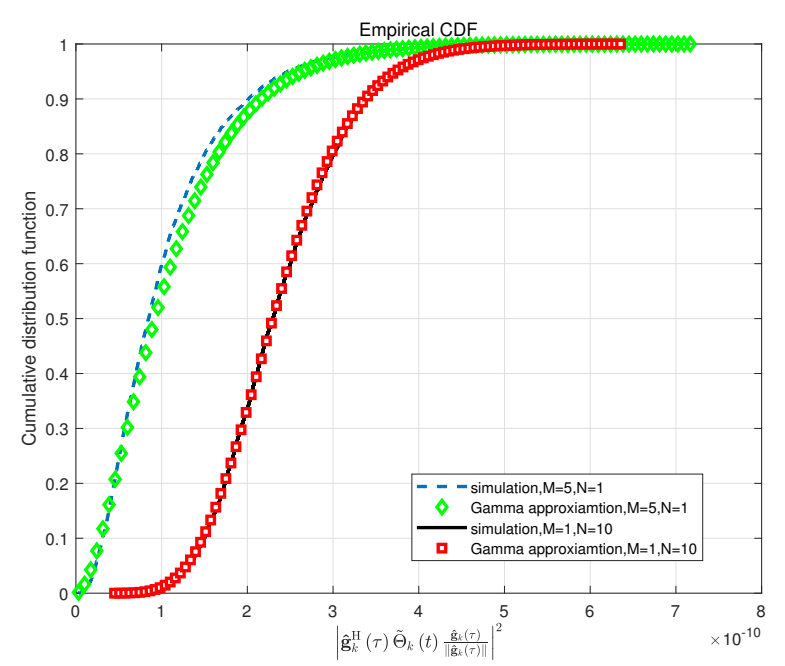

Figure 2. Cumulative distribution function of signal power with MRT precoder under different $M$ and $N$.

Based on the lemmas above, the spectral efficiency for downlink transmission with both MRT and ZF precoders under hardware impairments is analyzed. The theorems and corollary stated below give the closed-form expressions of the downlink achievable rates and system asymptotic performance.

Theorem 1. When MRT precoder is used, the closed-form expression of the downlink achievable rate under hardware impairments is given by

$$
R^{\mathrm{mrt}}(t)=\frac{D^{\mathrm{mrt}}(t)}{A^{\mathrm{mrt}}(t)+B^{\mathrm{mrt}}(t)+\xi^{\mathrm{UE}} / \rho_{\mathrm{dl}}}
$$

where

$$
\begin{aligned}
D^{\mathrm{mrt}}(t) & =\left(\frac{\Gamma\left(\chi_{k}^{\prime}(t)+\frac{1}{2}\right)}{\Gamma\left(\chi_{k}^{\prime}(t)\right)}\right)^{2} \theta_{k}^{\prime}(t), \\
\chi_{k}^{\prime}(t) & =N \frac{\left(\sum_{m=1}^{M} e^{-\left(\sigma_{\varphi, k}^{2}+\sigma_{\phi, m}^{2}\right)|t-\tau|} \beta_{m, k}^{2}(t)\right)^{2}}{\sum_{m=1}^{M} e^{-2\left(\sigma_{\varphi, k}^{2}+\sigma_{\phi, m}^{2}\right)|t-\tau|} \beta_{m, k}^{4}(t)}, \\
\theta_{k}^{\prime}(t) & =\frac{\sum_{m=1}^{M} e^{-2\left(\sigma_{\varphi, k}^{2}+\sigma_{\phi, m}^{2}\right)|t-\tau|} \beta_{m, k}^{4}(t)}{\sum_{m=1}^{M} e^{-\left(\sigma_{\varphi, k}^{2}+\sigma_{\phi, m}^{2}\right)|t-\tau|} \beta_{m, k}^{2}(t)} \\
A^{\mathrm{mrt}}(t) & =N \sum_{m=1}^{M} \beta_{m, k}^{2}(\tau)+\frac{1}{M} \sum_{m=1}^{M} \eta_{m, k}^{2}(\tau)-D^{\mathrm{mrt}}(t), \\
B^{\mathrm{mrt}}(t) & =\sum_{i \neq k}^{K} \frac{N \sum_{m=1}^{M} \beta_{m, i}^{2}(\tau) \lambda_{m, k}}{\theta_{i}(\tau)\left(\chi_{i}(\tau)-1\right)} .
\end{aligned}
$$

Proof of Theorem 1. Please refer to Appendix A. 
Theorem 2. When ZF precoder is used, the closed-form expression of the downlink achievable rate under hardware impairments is given by

$$
R^{\mathrm{zf}}(t)=\frac{D^{\mathrm{zf}}(t)}{A^{\mathrm{zf}}(t)+B^{\mathrm{zf}}(t)+\xi^{\mathrm{UE}} / \rho_{\mathrm{dl}}},
$$

where

$$
\begin{aligned}
D^{\mathrm{zf}}(t) & =\left(\frac{\Gamma\left(\kappa(t)+\frac{1}{2}\right)}{\Gamma(\kappa(t))}\right)^{2} \theta_{k}^{\prime}(t), \\
A^{\mathrm{zf}}(t) & =\frac{M N-K+1}{M} \sum_{m=1}^{M} \beta_{m, k}^{2}(\tau)-D^{\mathrm{zf}}(t), \\
B^{\mathrm{zf}}(t) & =\frac{K}{M} \sum_{m=1}^{M} \eta_{m, k}^{2}(\tau) \\
\kappa(t) & =\frac{M N-K+1}{M N} \chi_{k}^{\prime}(t) .
\end{aligned}
$$

Proof of Theorem 2. Please refer to Appendix B.

Then, in order to study the effects of phase noise further, we investigate a case where the number of antennas employed at each RAU goes infinity and the number of RAUs and users is fixed. The asymptotic performance provided in Corollary 1 is obtained based on (28) and (29).

Corollary 1. Let $N \rightarrow \infty$, the ultimate rate of user $k$ with both MRT and ZF precoders is given by

$$
R_{k}^{\infty}(t)=\frac{\sum_{m=1}^{M} e^{-\left(\sigma_{\varphi, k}^{2}+\sigma_{\phi, m}^{2}\right)|t-\tau|} \beta_{m, k}^{2}(\tau)}{\sum_{m=1}^{M} \beta_{m, k}^{2}(\tau)-\sum_{m=1}^{M} e^{-\left(\sigma_{\varphi, k}^{2}+\sigma_{\phi, m}^{2}\right)|t-\tau|} \beta_{m, k}^{2}(\tau)},
$$

Proof. Since the proof is similar for both precoders, we only provide the proof for MRT precoder. It can be seen that $\chi_{k}^{\prime}(t) \rightarrow \infty$ when the number of antennas $N \rightarrow \infty$. Therefore we can have $\lim _{N \rightarrow \infty}\left(\left(\frac{\Gamma\left(\chi_{k}^{\prime}(t)+\frac{1}{2}\right)}{\Gamma\left(\chi_{k}^{\prime}(t)\right)}\right)^{2}-\chi_{k}^{\prime}(t)\right)=0$ [29]. Then the limiting rate of user $k$ can be obtained directly by dividing the denominator and numerator of (28) by $N$. From Corollary 1 we can see that the ultimate rate without phase noise will be unlimited when $N$ tends to infinity, which means that phase noise limits the downlink spectral efficiency.

\section{Numerical Results}

In this section, a series of Monte Carlo simulations is used to verify the theoretical results obtained in Section 3. A circular single-cell massive MIMO system is considered. All of the RAUs and users are randomly distributed in the cell and the minimum access distance between RAUs and users is set as $r_{0}=30 \mathrm{~m}$. The channels are generated by (4), and other simulation parameters are presented in Table 1 .

Table 1. Basic simulation parameters.

\begin{tabular}{cc}
\hline Number of RAUs $\boldsymbol{M}$ & $\mathbf{5}$ \\
\hline Number of users $K$ & 2 \\
Cell radius $R$ & $1 \mathrm{~km}$ \\
Path loss exponent $\alpha$ & 3.7 \\
Power of uplink pilot symbol $\rho_{\mathrm{p}}$ & $1 \mathrm{Watt}$ \\
downlink transmit power $\rho_{\mathrm{dl}}$ & $10 \mathrm{Watts}$ \\
variance of thermal noise is assumed as $\sigma^{2}$ & $-174 \mathrm{dBm}$ \\
\hline
\end{tabular}


Figure 3 illustrates the theoretical and simulated spectral efficiency with MRT and ZF precoders versus the number of antennas per RAU. The spectral efficiency is the average rate between users. We assume that the variances of phase noise and amplified thermal noise are $\sigma_{\phi, m}^{2}=\sigma_{\varphi, k}^{2}=$ $1.58 \times 10^{-4} \forall m, k$ and $\xi^{\mathrm{UE}}=\xi^{\mathrm{BS}}=1.58 \sigma^{2} . t$ is set as $\tau+1$. It can be seen that the closed-form expressions in (28), (29) and the simulation results in (16) match well with each other. For both precoders, the spectral efficiency increases and gets more and more close to the limiting average rate with the increasing of $N$. When $N=100$, the system achieves $80 \%$ of the ultimate rate with ZF precoder and $76 \%$ with MRT precoder. Furthermore, it can be seen that ZF precoder achieves better performance than MRT.

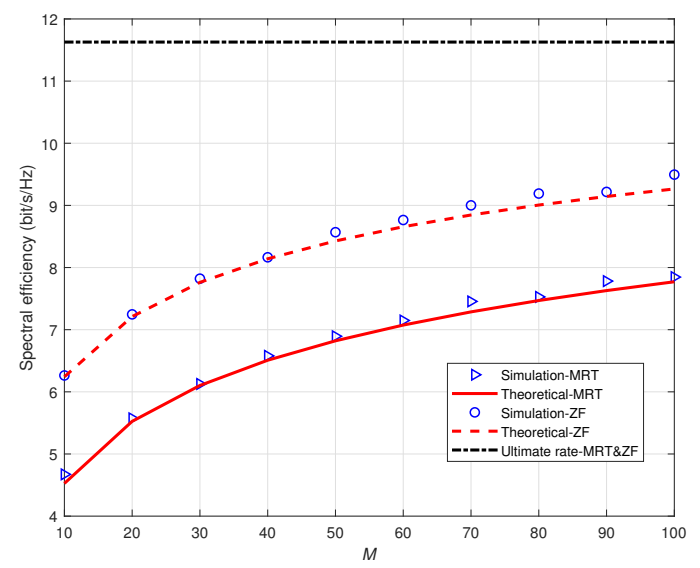

Figure 3. Spectral efficiency against $M$ with MRT and ZF precoders.

Next, we investigate the effects of phase noise. Figure 4 illustrates the theoretical spectral efficiency with MRT and ZF precoders against the variance of phase noise. Notably, the variance of phase noise reflects the strength of phase noise. It is assumed that the number of antennas $N=50$ and other system parameters have the same value as Figure 3. Figure 4 reveals that the spectral efficiency decreases monotonically with the variance of phase noise increasing. In addition, phase noise have a greater impact on ZF precoder. This results form the fact that ZF precoder is more sensitive to CSI. It can be noted that as the variance increases, the performance gap between MRT and ZF precoders becomes smaller. This is because when the phase noise is severe, the loss caused by unknown CSI at user side dominates rather than the interference between users.

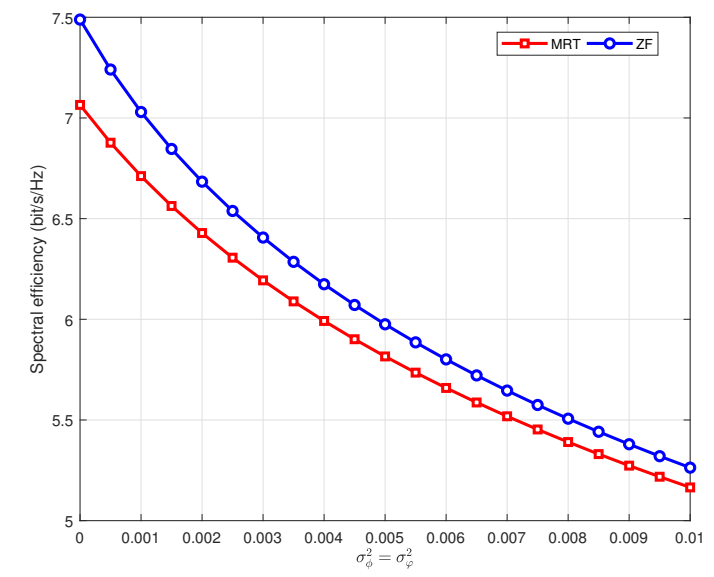

Figure 4. Spectral efficiency against $\sigma_{\phi}^{2}=\sigma_{\varphi}^{2}$ using MRT and ZF precoders with $\sigma_{\phi, m}^{2}=\sigma_{\phi, k}^{2}=\sigma_{\phi}^{2}=$ $\sigma_{\varphi}^{2}, \forall m, k$. 
Figure 5 illustrates the instantaneous spectral efficiency during the downlink transmission phase. The number of antennas employed at each RAU is assumed as $N=40$ and the variance of phase noise is set as $\sigma_{\phi, m}^{2}=\sigma_{\varphi, k}^{2}=1.58 \times 10^{-4} \forall m, k$. In addition, the coherence time of channel is set as $T=200$. As shown in Figure 5, the spectral efficiency degrades as $t$ increases. This is because the uncertainty of the phase drift between downlink transmission phase and pilot training phase increases with the growing of $t$. Figure 5 reveals that it's improper to use the estimated CSI obtained during the pilot phase for the whole data transmission phase.

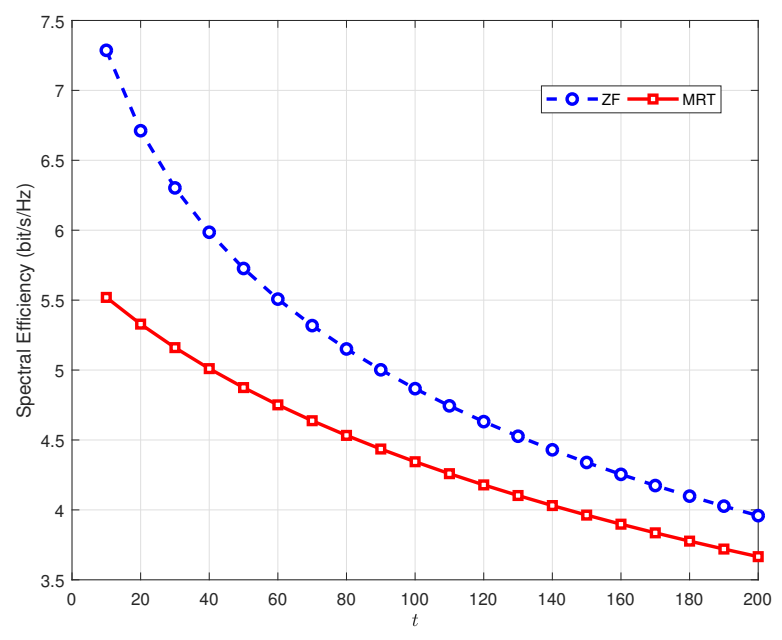

Figure 5. Spectral efficiency against $t$ with MRT and ZF precoders.

\section{Conclusions}

In this paper, we analyzed downlink spectral efficiency with hardware impairments in distributed massive MIMO. Initially, employing pilot symbol assisted transmission, we obtained the estimated CSI in a more realistic scenario where transmission is distorted by phase noise and amplified thermal noise. Next, we used the imperfect CSI to derive the closed-form expressions for downlink achievable rates with MRT and ZF precoders. In addition, we obtained the ultimate rate when $N \rightarrow \infty$. It can be seen that the rate performance was limited by phase noise. Then, numerical results proved that the theoretical analysis was accurate. Furthermore, they also revealed that ZF can achieve larger spectral efficiency than MRT precoder, and hardware impairments had a greater impact on ZF precoder. Finally, spectral efficiency degraded with the increasing of the variance of phase noise and downlink transmission time.

In the future work, we intend to extend our research considering a more effective phase noise model which could lead finer precoding strategies to improve the theoretical rates.

Author Contributions: Formal analysis, Q.L.; Supervision, J.L., P.Z., D.W. and X.Y.; Validation, Q.L.; Writing—original draft, Q.L.; Writing_review \& editing, Q.L. and J.L.

Funding: This work was supported in part by National Natural Science Foundation of China (NSFC) (Grant NO. 61501113, 61571120, 61871122), Jiangsu Provincial Natural Science Foundation (Grant No.BK20150630, BK20180011), six talent peaks project in Jiangsu province, and National Key Special Program No.2018ZX03001008-002.

Conflicts of Interest: The authors declare no conflict of interest.

\section{Appendix A. Proof of Theorem 1}

When MRT precoder is chosen, the following three terms $\left|\mathbb{E}\left[\mathbf{g}_{k}^{\mathrm{H}}(\tau) \tilde{\Theta}_{k}(t) \mathbf{w}_{k}\right]\right|^{2}, A(t)$ and $B(t)$ need to be calculated showing in (16). 
For the term $\left|\mathbb{E}\left[\mathbf{g}_{k}^{\mathrm{H}}(\tau) \tilde{\Theta}_{k}(t) \mathbf{w}_{k}\right]\right|^{2}$, we can obtain

$$
\begin{aligned}
& \left|\mathbb{E}\left[\mathbf{g}_{k}^{\mathrm{H}}(\tau) \tilde{\Theta}_{k}(t) \mathbf{w}_{k}\right]\right|^{2} \\
& \stackrel{(a)}{=}\left|\mathbb{E}\left[\mathbf{g}_{k}^{\mathrm{H}}(\tau) \mathbb{E}\left[\tilde{\Theta}_{k}(t)\right] \mathbf{w}_{k}\right]\right|^{2} \\
& \stackrel{(b)}{=}\left|\mathbb{E}\left[\left|\hat{\mathbf{g}}_{k}^{\mathrm{H}}(\tau) \mathbf{D}_{k, \tau}(t) \mathbf{w}_{k}\right|\right]\right|^{2} \\
& \stackrel{(c)}{=}\left(\frac{\Gamma\left(\chi_{k}^{\prime}(\tau)+\frac{1}{2}\right)}{\Gamma\left(\chi_{k}^{\prime}(\tau)\right)}\right)^{2} \theta_{k}^{\prime}(\tau),
\end{aligned}
$$

where $(a)$ is obtained because $\tilde{\Theta}_{k}(t)$ is independent of $\mathbf{g}_{k}(\tau)$ and $\mathbf{w}_{k}$. By exploiting the fact that $\mathbb{E}\left[e^{-j\left(\theta_{k}^{m}(t)-\theta_{k}^{m}(\tau)\right)}\right]=e^{-\frac{\sigma_{\varphi, k}^{2}+\sigma_{\phi, m}^{2}}{2}|t-\tau|}$ and $\mathbf{e}_{k}(t)$ is independent of $\mathbf{w}_{k}$, we can get $(b)$, and $(c)$ results from Lemma 2, Lemma 3 and the relationship between Gamma distribution and Nakagami distribution.

For the term $A(t)$, we obtain

$$
\begin{aligned}
& \operatorname{var}\left(\mathbf{g}_{k}^{\mathrm{H}}(\tau) \tilde{\Theta}_{k}(t) \mathbf{w}_{k}\right) \\
& =\mathbb{E}\left[\left|\hat{\mathbf{g}}_{k}^{\mathrm{H}}(\tau) \tilde{\Theta}_{k}(t) \mathbf{w}_{k}\right|^{2}\right]+\mathbb{E}\left[\left|\mathbf{e}_{k}^{\mathrm{H}}(\tau) \tilde{\Theta}_{k}(t) \mathbf{w}_{k}\right|^{2}\right] \\
& -\left|\mathbb{E}\left[\mathbf{g}_{k}^{\mathrm{H}}(\tau) \tilde{\Theta}_{k}(t) \mathbf{w}_{k}\right]\right|^{2} .
\end{aligned}
$$

The first term of (A2) can be calculated as

$$
\mathbb{E}\left[\left|\hat{\mathbf{g}}_{k}^{\mathrm{H}}(\tau) \tilde{\Theta}_{k}(t) \frac{\hat{\mathbf{g}}_{k}(\tau)}{\left\|\hat{\mathbf{g}}_{k}(\tau)\right\|}\right|^{2}\right] \stackrel{(a)}{=} N \sum_{m=1}^{M} \beta_{m, k}^{2}(\tau),
$$

where (a) results from (26).

Next the second term can be given by

$$
\mathbb{E}\left[\left|\mathbf{e}_{k}^{\mathrm{H}}(\tau) \tilde{\Theta}_{k}(t) \frac{\hat{\mathbf{g}}_{k}(\tau)}{\left\|\hat{\mathbf{g}}_{k}(\tau)\right\|}\right|^{2}\right] \stackrel{(a)}{=} \frac{1}{M} \sum_{m=1}^{M} \eta_{m, k}^{2}(\tau),
$$

where $(a)$ can be obtained by exploiting the fact that $\left\|\mathbf{e}_{k}^{\mathrm{H}}(\tau) \tilde{\Theta}_{k}(t)\right\|^{2} \sim \Gamma\left(\chi_{\mathrm{e}}(\tau), \theta_{\mathrm{e}}(\tau)\right)$ and (25).

For the last term $B(t)$, we first calculate

$$
\begin{aligned}
\mathbb{E}\left[\frac{1}{\left\|\hat{\mathbf{g}}_{i}\right\|^{2}}\right] & \stackrel{(a)}{=} \int_{0}^{\infty} \frac{1}{x} x^{\chi_{i}-1} \frac{e^{-x / \theta_{i}}}{\theta_{i}^{\chi_{i}} \Gamma\left(\chi_{i}\right)} d x \\
& =\frac{1}{\theta_{i}^{\chi_{i}} \Gamma\left(\chi_{i}\right)} \theta_{i}^{\chi_{i}-1} \Gamma\left(\chi_{i}-1\right) \\
& =\frac{1}{\theta_{i}\left(\chi_{i}-1\right)}
\end{aligned}
$$


where (a) results from Remark 2 and we omit $(t)$ in (A5). Based on (A5), we have

$$
\begin{aligned}
& \mathbb{E}\left[\left|\mathbf{g}_{k}^{\mathrm{H}}(\tau) \tilde{\Theta}_{k}(t) \mathbf{w}_{i}\right|^{2}\right] \\
& \stackrel{(a)}{\asymp} \mathbb{E}\left[\left|\mathbf{g}_{k}^{\mathrm{H}}(\tau) \tilde{\Theta}_{k}(t) \hat{\mathbf{g}}_{i}(\tau)\right|^{2}\right] \mathbb{E}\left[\frac{1}{\left\|\hat{\mathbf{g}}_{i}(\tau)\right\|^{2}}\right] \\
& =\frac{\mathbb{E}\left[\sum_{m=1}^{M} \beta_{m, i}^{2}(\tau) \lambda_{m, k} \hat{\mathbf{h}}_{m, k}^{\mathrm{H}} \hat{\mathbf{h}}_{m, k}\right]}{\theta_{i}(\tau)\left(\chi_{i}(\tau)-1\right)} \\
& =\frac{N \sum_{m=1}^{M} \beta_{m, i}^{2}(\tau) \lambda_{m, k}}{\theta_{i}(\tau)\left(\chi_{i}(\tau)-1\right)},
\end{aligned}
$$

where $x \asymp y$ means $\lim _{N \rightarrow \infty}(x-y)=0,(a)$ results from Lemma 4 (ii) of [32].

Finally, combining (A1)-(A6) concludes the proof.

\section{Appendix B. Proof of the Theorem 2}

To derive the closed-form expression of (16) with ZF precoder, the following three terms $\left|\mathbb{E}\left[\mathbf{g}_{k}^{\mathrm{H}}(\tau) \tilde{\Theta}_{k}(t) \mathbf{w}_{k}\right]\right|^{2}, A(t)$ and $B(t)$ need to be calculated, wherein $\mathbf{w}_{k}=\frac{\mathbf{a}_{k}(\tau)}{\left\|\mathbf{a}_{k}(\tau)\right\|}$.

For the term $\left|\mathbb{E}\left[\mathbf{g}_{k}^{\mathrm{H}}(\tau) \tilde{\Theta}_{k}(t) \mathbf{w}_{k}\right]\right|^{2}$, we obtain

$$
\begin{aligned}
& \left|\mathbb{E}\left[\mathbf{g}_{k}^{\mathrm{H}}(\tau) \tilde{\Theta}_{k}(t) \mathbf{w}_{k}\right]\right|^{2} \\
& \stackrel{(a)}{=}\left|\mathbb{E}\left[\mathbf{g}_{k}^{\mathrm{H}}(\tau) \mathbb{E}\left[\tilde{\Theta}_{k}(t)\right] \mathbf{w}_{k}\right]\right|^{2} \\
& \stackrel{(b)}{=}\left|\mathbb{E}\left[\hat{\mathbf{g}}_{k}^{\mathrm{H}}(\tau) \mathbf{D}_{k, \tau}(t) \frac{\mathbf{a}_{k}(\tau)}{\left\|\mathbf{a}_{k}(\tau)\right\|}\right]\right|^{2} \\
& \stackrel{(c)}{=}\left(\frac{\Gamma\left(\kappa(t)+\frac{1}{2}\right)}{\Gamma(\kappa(t))}\right)^{2} \theta_{k}^{\prime}(t),
\end{aligned}
$$

where $(a)$ is obtained because $\tilde{\Theta}_{k}(t)$ is independent of $\mathbf{g}(\tau)$ and $\mathbf{w}_{k} .(b)$ is obtained due to the independence of $\mathbf{e}_{k}(\tau)$ and $\mathbf{w}_{k}$. (c) results from Lemma 2, Lemma 3 and the relationship between Gamma distribution and Nakagami distribution.

Similar to the proof of Theorem 1, to get the closed-form expression of $A(t)$, we need to calculate the following two terms

$$
\begin{aligned}
& \mathbb{E}\left[\left|\hat{\mathbf{g}}_{k}^{\mathrm{H}}(\tau) \tilde{\Theta}_{k}(t) \frac{\mathbf{a}_{k}(\tau)}{\left\|\mathbf{a}_{k}(\tau)\right\|}\right|^{2}\right], \\
& \mathbb{E}\left[\left|\mathbf{e}_{k}^{\mathrm{H}}(\tau) \tilde{\Theta}_{k}(t) \frac{\mathbf{a}_{k}(\tau)}{\left\|\mathbf{a}_{k}(\tau)\right\|}\right|^{2}\right] .
\end{aligned}
$$

The first term (A8) can be given by

$$
\mathbb{E}\left[\left|\hat{\mathbf{g}}_{k}^{\mathrm{H}}(\tau) \tilde{\Theta}_{k}(t) \frac{\mathbf{a}_{k}(\tau)}{\left\|\mathbf{a}_{k}(\tau)\right\|}\right|^{2}\right]=\frac{M N-K+1}{M} \sum_{m=1}^{M} \beta_{m, k}^{2}(\tau),
$$

which results from (27). 
Next the second term can be given by

$$
\mathbb{E}\left[\left|\mathbf{e}_{k}^{\mathrm{H}}(\tau) \tilde{\Theta}_{k}(t) \frac{\mathbf{a}_{k}(\tau)}{\left\|\mathbf{a}_{k}(\tau)\right\|}\right|^{2}\right]=\frac{1}{M} \sum_{m=1}^{M} \eta_{m, k}^{2}(\tau),
$$

which results from (25) and the fact that $\left\|\mathbf{e}_{k}^{\mathrm{H}}(\tau) \tilde{\Theta}_{k}(t)\right\|^{2} \sim \Gamma\left(\chi_{\mathrm{e}}(\tau), \theta_{\mathrm{e}}(\tau)\right)$.

For the term $B(t)$, we can have

$$
\begin{aligned}
& \mathbb{E}\left[\left|\mathbf{g}_{k}^{\mathrm{H}}(\tau) \tilde{\Theta}_{k}(t) \mathbf{w}_{i}\right|^{2}\right] \\
& \stackrel{(a)}{=} \mathbb{E}\left[\left|\mathbf{e}_{k}^{\mathrm{H}}(\tau) \tilde{\Theta}_{k}(t) \mathbf{w}_{i}\right|^{2}\right] \\
& =\frac{1}{M} \sum_{m=1}^{M} \eta_{m, k}^{2}(\tau),
\end{aligned}
$$

where $(a)$ results from the property of ZF precoder.

Substituting (A7) and (A10)-(A12) into (16) completes the proof.

\section{References}

1. Wang, J.; Zhu, H.; Gomes, N.J.; Wang, J. Frequency Reuse of Beam Allocation for Multiuser Massive MIMO Systems. IEEE Trans. Wirel. Commun. 2018, 17, 2346-2359. [CrossRef]

2. Wang, C.X.; Haider, F.; Gao, X.; You, X.H.; Yang, Y.; Yuan, D.; Aggoune, H.; Haas, H.; Fletcher, S.; Hepsaydir, E. Cellular architecture and key technologies for $5 \mathrm{G}$ wireless communication networks. IEEE Commun. Mag. 2014, 52, 122-130. [CrossRef]

3. Jiang, F.; Chen, J.; Swindlehurst, A.L.; López-Salcedo, J.A. Massive MIMO for Wireless Sensing with a Coherent Multiple Access Channel. IEEE Trans. Signal Process. 2015, 63, 3005-3017. [CrossRef]

4. Ciuonzo, D.; Rossi, P.S.; Dey, S. Massive MIMO Channel-Aware Decision Fusion. IEEE Trans. Signal Process. 2015, 63, 604-619. [CrossRef]

5. Ciuonzo, D.; Rossi, P.S.; Dey, S. Massive MIMO meets decision fusion: Decode-and-fuse vs. decode-then-fuse. In Proceedings of the 2014 IEEE 8th Sensor Array and Multichannel Signal Processing Workshop (SAM), A Coruna, Spain, 22-25 June 2014; pp. 265-268. [CrossRef]

6. Shirazinia, A.; Dey, S.; Ciuonzo, D.; Rossi, P.S. Massive MIMO for Decentralized Estimation of a Correlated Source. IEEE Trans. Signal Process. 2016, 64, 2499-2512. [CrossRef]

7. Cao, J.; Wang, D.; Li, J.; Sun, Q.; Hu, Y. Uplink spectral efficiency analysis of multi-cell multi-user massive MIMO over correlated Ricean channel. Sci. China Inf. Sci. 2018, 61, 082305. [CrossRef]

8. Björnson, E.; Matthaiou, M.; Debbah, M. Massive MIMO with non-ideal arbitrary arrays: Hardware scaling laws and circuit-aware design. IEEE Trans. Wirel. Commun. 2015, 14, 4353-4368. [CrossRef]

9. Dai, L. An Uplink Capacity Analysis of the Distributed Antenna System (DAS): From Cellular DAS to DAS with Virtual Cells. IEEE Trans. Wirel. Commun. 2014, 13, 2717-2731.

10. Liu, Z.; Dai, L. On the Scaling Behavior of Average Ergodic Capacity of Distributed MIMO Systems. In Proceedings of the 2015 IEEE Global Communications Conference (GLOBECOM), San Diego, CA, USA, 6-10 December 2015; pp. 1-6. [CrossRef]

11. Wang, J.; Dai, L. Asymptotic Rate Analysis of Downlink Multi-User Systems with Co-Located and Distributed Antennas. IEEE Trans. Wirel. Commun. 2015, 14, 3046-3058. [CrossRef]

12. Wang, J.; Dai, L. Downlink Rate Analysis for Virtual-Cell Based Large-Scale Distributed Antenna Systems. IEEE Trans. Wirel. Commun. 2016, 15, 1998-2011. [CrossRef]

13. Khadka, A.; Adachi, K.; Sun, S.; Wang, J.; Zhu, H.; Wang, J. Cooperative Transmission Strategy over Users' Mobility for Downlink Distributed Antenna Systems. In Proceedings of the GLOBECOM 2017-2017 IEEE Global Communications Conference, Singapore, 4-8 December 2017; pp. 1-6. [CrossRef]

14. Zhu, H.; Wang, J. Radio Resource Allocation in Multiuser Distributed Antenna Systems. IEEE J. Sel. Areas Commun. 2013, 31, 2058-2066. [CrossRef] 
15. Lv, Q.; Li, J.; Zhu, P.; You, X. Spectral Efficiency Analysis for Bidirectional Dynamic Network with Massive MIMO Under Imperfect CSI. IEEE Access 2018, 6, 43660-43671. [CrossRef]

16. Studer, C.; Wenk, M.; Burg, A. MIMO transmission with residual transmit-RF impairments. In Proceedings of the 2010 International ITG Workshop on Smart Antennas (WSA), Bremen, Germany, 23-24 February 2010; pp. 189-196. [CrossRef]

17. Papazafeiropoulos, A.; Clerckx, B.; Ratnarajah, T. Mitigation of phase noise in massive MIMO systems: A rate-splitting approach. In Proceedings of the 2017 IEEE International Conference on Communications (ICC), Paris, France, 21-25 May 2017; pp. 1-7. [CrossRef]

18. Pitarokoilis, A.; Mohammed, S.K.; Larsson, E.G. Uplink Performance of Time-Reversal MRC in Massive MIMO Systems Subject to Phase Noise. IEEE Trans. Wirel. Commun. 2015, 14, 711-723. [CrossRef]

19. Pitarokoilis, A.; Mohammed, S.K.; Larsson, E.G. Achievable rates of ZF receivers in massive MIMO with phase noise impairments. In Proceedings of the 2013 Asilomar Conference on Signals, Systems and Computers, Pacific Grove, CA, USA, 3-6 November 2013; pp. 1004-1008. [CrossRef]

20. Li, J.; Wang, D.; Zhu, P.; You, X. Uplink Spectral Efficiency Analysis of Distributed Massive MIMO with Channel Impairments. IEEE Access 2017, 5, 5020-5030. [CrossRef]

21. Fang, Y.; Li, X.; Qiu, L. Asymptotic Equivalent Performance of Uplink Massive MIMO Systems with Phase Noise. In Proceedings of the 2018 IEEE International Conference on Communications (ICC), Kansas City, MO, USA, 20-24 May 2018; pp. 1-6. [CrossRef]

22. Zhu, J.; Schober, R.; Bhargava, V.K. Physical layer security for massive MIMO systems impaired by phase noise. In Proceedings of the 2016 IEEE 17th International Workshop on Signal Processing Advances in Wireless Communications (SPAWC), Edinburgh, UK, 3-6 July 2016; pp. 1-5. [CrossRef]

23. Corvaja, R.; Armada, A.G. Phase Noise Degradation in Massive MIMO Downlink with Zero-Forcing and Maximum Ratio Transmission Precoding. IEEE Trans. Veh. Technol. 2016, 65, 8052-8059. [CrossRef]

24. Krishnan, R.; Khanzadi, M.R.; Krishnan, N.; Wu, Y.; i Amat, A.G.; Eriksson, T.; Schober, R. Linear Massive MIMO Precoders in the Presence of Phase Noise - A Large-Scale Analysis. IEEE Trans. Veh. Technol. 2016, 65, 3057-3071. [CrossRef]

25. Petrovic, D.; Rave, W.; Fettweis, G. Effects of Phase Noise on OFDM Systems with and without PLL: Characterization and Compensation. IEEE Trans. Commun. 2007, 55, 1607-1616. [CrossRef]

26. Papazafeiropoulos, A.; Clerckx, B.; Ratnarajah, T. Rate-splitting to mitigate residual transceiver hardware impairments in massive MIMO systems. IEEE Trans. Veh. Technol. 2017, 66, 8196-8211. [CrossRef]

27. Li, J.; Wang, D.; Zhu, P.; You, X. Spectral efficiency analysis of single-cell multi-user large-scale distributed antenna system. IET Commun. 2014, 8, 2213-2221. [CrossRef]

28. Li, J.; Wang, D.; Zhu, P.; Wang, J.; You, X. Downlink Spectral Efficiency of Distributed Massive MIMO Systems with Linear Beamforming Under Pilot Contamination. IEEE Trans. Veh. Technol. 2018, 67, 1130-1145. [CrossRef]

29. Jose, J.; Ashikhmin, A.; Marzetta, T.L.; Vishwanath, S. Pilot contamination and precoding in multi-cell TDD systems. IEEE Trans. Wirel. Commun. 2011, 10, 2640-2651. [CrossRef]

30. Hosseini, K.; Yu, W.; Adve, R.S. Large-scale MIMO versus network MIMO for multicell interference mitigation. In Proceedings of the 2014 IEEE 15th International Workshop on Signal Processing Advances in Wireless Communications (SPAWC), Toronto, ON, Canada, 22-25 June 2014; pp. 70-74. [CrossRef]

31. Li, J.; Wang, D.; Zhu, P.; You, X. Benefits of Beamforming Training Scheme in Distributed Large-Scale MIMO Systems. IEEE Access 2018, 6, 7432-7444. [CrossRef]

32. Hoydis, J.; ten Brink, S.; Debbah, M. Massive MIMO in the UL/DL of Cellular Networks: How Many Antennas Do We Need? IEEE J. Sel. Areas Commun. 2013, 31, 160-171. [CrossRef]

(C) 2018 by the authors. Licensee MDPI, Basel, Switzerland. This article is an open access article distributed under the terms and conditions of the Creative Commons Attribution (CC BY) license (http:/ / creativecommons.org/licenses/by/4.0/). 